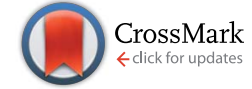

Cite this: J. Anal. At. Spectrom., 2015, 30,666

\title{
Evidence for an unorthodox firing sequence employed by the Berlin Painter: deciphering ancient ceramic firing conditions through high- resolution material characterization and replication
}

\author{
I. Cianchetta, ${ }^{\text {*a }}$ K. Trentelman, ${ }^{\text {a }}$ J. Maish, ${ }^{\text {b D. Saunders, }}{ }^{\text {b } B . ~ F o r a n, ~}{ }^{c}$ M. Walton, ${ }^{d}$ \\ Ph. Sciau, ${ }^{e}$ T. Wang, ${ }^{e}$ E. Pouyet, ${ }^{f}$ M. Cotte, ${ }^{f g}$ F. Meirer, ${ }^{\text {h }}$ Y. Liu, ${ }^{i}$ P. Pianetta' and A. Mehta
}

\begin{abstract}
XANES spectroscopy was used to complement the results previously obtained with Raman spectroscopy by the same group to determine the firing conditions used in the production of a single vessel painted by the Berlin Painter in the 5th century B.C. The vessel, part of the collection of the J. Paul Getty Museum, presents a complicated layered architecture of black and red gloss, with different stratigraphies present on the interior and exterior surfaces. The study of two samples, one each from the interior and exterior surface of the vessel, was performed with the complementary analytical techniques of X-ray nano- and microspectroscopy (X-ray fluorescence spectroscopy (XRF) and full-field transmission $X$-ray microspectroscopy (FF-XANES) across the Fe $\mathrm{K}$ edge), and supported by a replication study. The replicates, made in a laboratory furnace providing complete control over the firing temperature and oxygen partial pressure, provided a paradigm for the comparison of the mineralogical phases observed in the ancient samples, which led to a deeper understanding of the firing conditions necessary for the production of the Berlin Painter's vessel. Our results confirm the necessity of multiple firings and painting applications to obtain the Berlin Painter's architecture and provide a further example of the multiplicity of techniques and practices employed by the potters of the Kerameikos in ancient Athens.
\end{abstract}

Received 30th October 2014 Accepted 9th December 2014 DOI: $10.1039 / c 4 j a 00376 d$

www.rsc.org/jaas

\section{Introduction}

Athenian pottery, the ceramics produced in the Attica region of Greece between the 6th and 4th centuries B.C., represents a technological benchmark of antiquity. For decades, material scientists and archaeologists have studied these black-figure and red-figure vessels ${ }^{\mathbf{1 - 1 4}}$ (with figures painted directly in black gloss, or left in reserve, respectively), but aspects of the painting practices and firing conditions by which they were produced are still under debate. Together with black gloss, a red gloss, frequently referred to as Coral Red, is also sometimes found. ${ }^{9}$ Decorations and background washes were painted on the clay body with a dispersion of Fe-rich illitic clay, refined through the levigation process, in which the carbonates and larger particles

${ }^{a}$ Getty Conservation Institute, Los Angeles, CA, USA. E-mail: icianchetta@getty.edu ${ }^{b} J$. Paul Getty Museum, Los Angeles, CA, USA

${ }^{c}$ Aerospace Corporation, El Segundo, CA, USA

${ }^{d}$ Northwestern University, Evanston, IL, USA

${ }^{e}$ CEMES, CNRS, Université de Toulouse, Toulouse, France ${ }^{f}$ ESRF, Grenoble, France

${ }^{g}$ Laboratoire d'archéologie moléculaire et structurale, LAMS, CNRS, UMR 8220, Paris, France

${ }^{h}$ University of Utrecht, Utrecht, Nederland

${ }^{i}$ Stanford Synchrotron Radiation Lightsource, Palo Alto, CA, USA are settled out from the clay, and the resulting fine fraction is collected. ${ }^{2,8,15}$ The painted areas transform into vitrified slips during firing, with the colour determined by the oxidation state of the iron: black, produced by $\mathrm{Fe}^{2+}$ (in magnetite, $\mathrm{Fe}_{3} \mathrm{O}_{4}$, and hercynite $\mathrm{FeAl}_{2} \mathrm{O}_{4}$ ), is responsible for the black colour, while red is produced by $\mathrm{Fe}^{3+}$ (in hematite, $\alpha-\mathrm{Fe}_{2} \mathrm{O}_{3}$, and maghemite, $\gamma-\mathrm{Fe}_{2} \mathrm{O}_{3}$ ). Ancient potters had a sophisticated control over the firing temperature and oxygen fugacity, and thus were able to selectively reduce or oxidize different decorative elements on the vessels to create the striking red and black motifs characteristic of this production.

It has long been believed that the firing protocol for the production of all Athenian pottery was a single three-stage firing employing alternating oxidizing-reducing-oxidizing conditions in the kiln..$^{1-3,14-17}$ Our study into the composition, morphology and mineralogy of red and black gloss is building a picture that suggests this is an oversimplified view. Our study on Coral Red ${ }^{9}$ revealed that while, in some vessels, the red and black gloss had sufficiently different compositions that may have allowed them to be produced together in a single firing, in other vessels multiple, separate, firings must have been employed. Another study, in which different applications of black gloss on a single vessel fragment were compared, again suggested that multiple rounds of painting/firing were employed. ${ }^{10}$ 
In this paper we continue to explore whether the single threestage firing model is adequate to produce vessels decorated with both black gloss and red gloss. The object under investigation is the neck of a red-figure hydria in the collection of the J. Paul Getty Museum (JPGM 81.AE.206.d.2005), shown in Fig. 1, attributed to the Berlin Painter (active in the early fifth century B.C.). We employed multiple complementary probes to perform detailed high resolution material characterization of the chemistry, morphology, and nanoscale mineralogy of the vessel, supplemented with the study of replicates created under firing conditions achievable in a large wood-fired kiln to decipher the precise firing protocol that must have been used to create this piece. This work, taken together with our earlier Raman work on this piece produced by one of the master craftsmen, is invaluable in giving us an in-depth glimpse into the state-of-the-art of black gloss technology.

\section{Background}

Iron exists in several different mineralogical forms in clay and many of these minerals are found in Fe-rich pottery. There is evidence of trace amounts of Fe silicate, and even metallic $\mathrm{Fe}^{18}$ in ancient pottery, but iron oxides are the key minerals of interest. They include, hematite $\left(\alpha-\mathrm{Fe}_{2} \mathrm{O}_{3}\right)$, maghemite $\left(\gamma-\mathrm{Fe}_{2} \mathrm{O}_{3}\right), \mathrm{Fe}_{3} \mathrm{O}_{4}$ (magnetite), the less common $\epsilon-$ and $\beta-\mathrm{Fe}_{2} \mathrm{O}_{3}$, and $\mathrm{Fe}_{1-x} \mathrm{O}$ (wüstite). In clay containing $\mathrm{Al}$ (and sometimes $\mathrm{Ti}$ and $\mathrm{Mg}$ ), and fired under reducing conditions, $\mathrm{FeAl}_{2} \mathrm{O}_{4}$ (hercynite) is common. A recent investigation ${ }^{19}$ of the stability of iron oxides concluded that under mildly oxidizing conditions, in the presence of water, goethite $(\mathrm{FeOOH})$ and ferrihydrite $\left(\mathrm{Fe}(\mathrm{OH})_{3}\right)$ form, but under dry conditions maghemite $\left(\gamma-\mathrm{Fe}_{2} \mathrm{O}_{3}\right)$ or hematite $\left(\alpha-\mathrm{Fe}_{2} \mathrm{O}_{3}\right)$ are common. If the crystallites are nanocrystalline, maghemite is more stable than hematite. ${ }^{20}$ Furthermore, oxidation of magnetite or hercynite (both spinels) first transforms into maghemite (also a spinel); in fact the process of topotactic oxidation of magnetite is commonly referred to in the literature as maghemitization. ${ }^{21,22}$ Upon prolonged oxidation, maghemite grains slowly grow, and eventually undergo a structural transformation into hematite. ${ }^{23}$ And finally, there is some recent preliminary evidence that at moderate temperatures goethite transforms into maghemite. ${ }^{24}$ In a ceramic sherd, the distribution of maghemite in relation to hercynite/magnetite, on one hand, and hematite, on the other hand, is crucial in deciphering the firing conditions used in its production. In fact, based on the above discussion on the stability and transformation pathways of Fe minerals, every step in the production of black gloss on a red body can be outlined in terms of occurrence and transformation of Fe mineralogy. Transformation pathways can be inferred through the analysis of the intensity and pre-edge features of Fe XANES spectra, ${ }^{17,25}$ but the statistics of the preedge features in our FF-XANES data did not allow us to adopt this method.

An earlier study ${ }^{26}$ by our group on this same vessel fragment painted by the Berlin Painter employed Raman spectroscopy to characterize the layers. The intensity, position and width of the Raman $\mathrm{E}_{\mathrm{g}}$ band of hematite at $300 \mathrm{~cm}^{-1}$ can provide information regarding the firing temperature. ${ }^{27}$ Comparing spectra
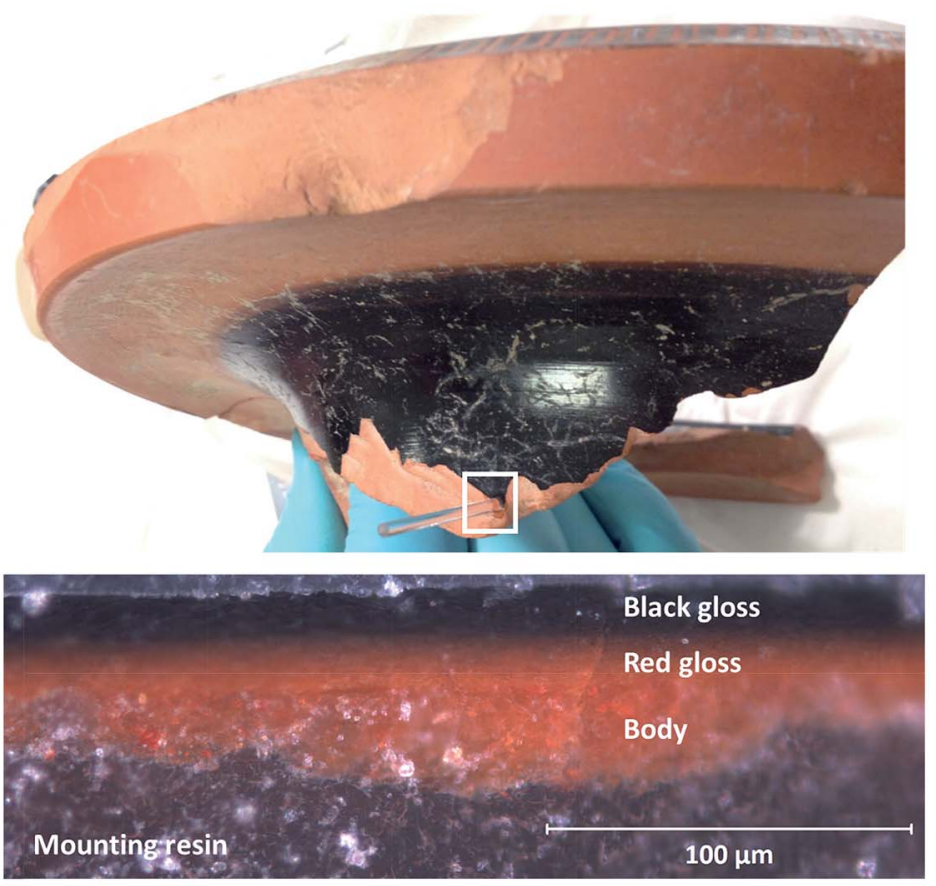

a)

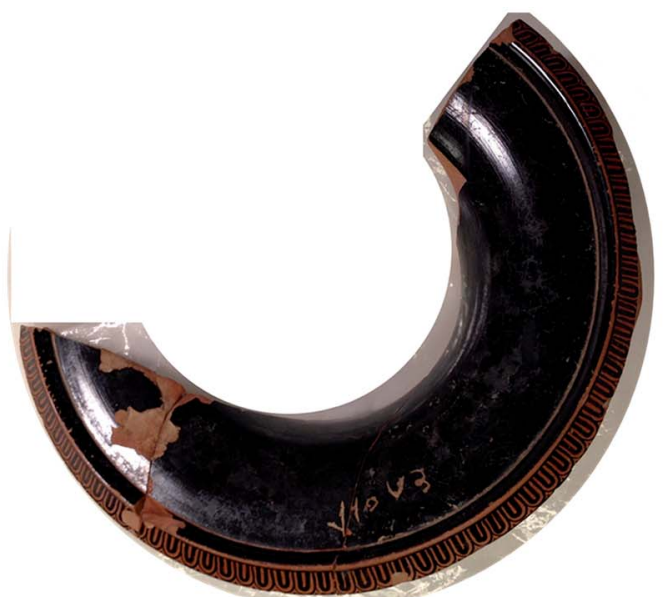

c)

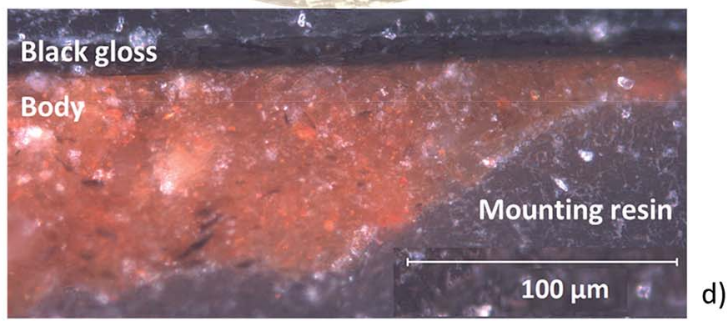

Fig. 1 (a) Sampling point of JPGM 81.AE.206.d.2005_ext. (b) Cross-section of sample JPGM 81.AE.206.d.2005_ext. From top: black gloss, red gloss, body. (c) Sampling point of JPGM 81.AE.206.d.2005_int. (d) Cross-section of sample JPGM 81.AE.206.d.2005_int. From top: black gloss, body. 
from the red glossy layer and body of the ancient sherd to those obtained on replicate sherds fired under controlled temperature/atmospheric conditions, the results suggested a complex firing scheme was employed in the production of the ancient vessel. Specifically, the data demonstrated that two applications of refined clay and two separate firings were necessary to produce the observed complex architecture. First, a homogeneous application of clay was applied over the surface of the vessel, which was then fired at high temperature, under oxidizing conditions, to create the even red gloss layer found underneath the black gloss and also on the outer surface of the neck (see Fig. 1). Second, the decorative motif (painted areas intended to be black) was applied and the vessel subjected to a three-stage (oxidation-reduction-oxidation) firing, with the temperature of each stage successively lower than the previous to insure the retention of the desired vitrified layers.

Raman spectroscopy, however, does not have equal sensitivity to all the mineralogical phases that might be present in ceramics. ${ }^{7,18,28-31}$ In particular, although numerous diffraction studies ${ }^{7,32-35}$ have reported the presence of hercynite in ceramics, the detection of pure hercynite with Raman spectroscopy has never been reported in the characterization of ancient pottery. The Raman spectrum of hercynite included in the RUFF project database $^{36}$ is the only published spectrum found. Therefore, whether or not hercynite is present in ceramics cannot be determined by Raman spectroscopy. Raman spectroscopy also has lower sensitivity to maghemite compared to hematite. Since the presence of the $\gamma$-phase of $\mathrm{Fe}^{3+}$ is diagnostic of the reoxidation of $\mathrm{Fe}^{2+}$, and thus of critical importance for the determination of the firing conditions, additional techniques must be employed to ensure detection of all mineralogical phases.

The study presented here describes the use of X-ray absorption near edge structure (XANES) spectroscopy to more completely characterize the iron species present in the glossy slips and the body clay of ancient ceramics. XANES is able to fully characterize the presence of hercynite, maghemite and hematite, providing additional insights into the firing conditions employed in their production. The identification of magnetite can be challenging since the dominant spectral contrast in the Fe K edge X-ray absorption spectrum between pure magnetite and an equal proportion mixture of hercynite $\left(\mathrm{Fe}^{2+}\right) /$ hematite $\left(\mathrm{Fe}^{3+}\right)$ or hercynite $\left(\mathrm{Fe}^{2+}\right) /$ maghemite $\left(\mathrm{Fe}^{3+}\right)$ is in the pre-edge features. ${ }^{37,38}$ Hence, the use of both Raman spectroscopy and XANES allows a complete characterization of the materials present in the ceramic slips, allowing the firing conditions used in their creation to be inferred.

\section{Experimental}

\section{Samples}

Berlin Painter sherd. Two samples, one each from the exterior and the interior (just beneath the overhanging lip) of the sherd were taken from the locations indicated in Fig. 1a and c, respectively. Optical examination of the cross-section of the sample taken from the exterior (named JPGM 81.AE.206.d.2005_ext in Fig. 1b, henceforth referred to as EXT) clearly shows a layered structure: a black gloss slip on top of a red glossy slip, both painted over the red body. Whereas the cross-section of the sample taken from the interior (named JPGM 81.AE.206.d.2005_int in Fig. 1d, henceforth referred to as INT), appears to show only two layers: the black gloss on top of the red body.

Replicate sherds. Replicate sherds (tiles approximately $2 \times 2$ $\mathrm{cm}$ ) were made from raw commercial illite clay (Redart clay, Resco Products Inc., Pittsburgh PA). This choice is compatible with previous studies ${ }^{\mathbf{1 5}, 39-42}$ that have identified illite as the type of clay used for the production of Greek ceramics. For the slip, the clay, mixed with 0.25 wt\% of $\mathrm{Na}_{2}\left(\mathrm{SiO}_{2}\right)_{n} \mathrm{O}$ (Caroline) and $\mathrm{NaCO}_{3}$ (Aldrich), was diluted until a specific gravity of 1.2 was reached and then allowed to settle for 20 hours. The supernatant was collected, dried and used in a dispersion to paint the tiles. In his replication experiments of Attic black gloss, Noble ${ }^{2}$ used sodium hexametaphosphate $\left(\mathrm{Na}_{6} \mathrm{P}_{6} \mathrm{O}_{18}\right)$ in order to obtain the fine fraction; potash (potassium carbonate, $\mathrm{K}_{2} \mathrm{CO}_{3}$ ) was similarly employed. In this work we used sodium silicate and sodium carbonate, deflocculating agents that are commonly used among the pottery community for the preparation of clay to be used for slips. The replicate sherds were fired in a quartz tube electric furnace (GSL 1600x, MTI Corporation) equipped with a PID temperature control via SCR power control and oxygen sensor (DS oxygen probe, Australian Oxytrol Systems Pty. Ltd). For the first oxidative stage $\left(T_{1}\right)$, two sets of replicate sherds were prepared: the temperature was increased at the rate of $12{ }^{\circ} \mathrm{C} \mathrm{min}$ m $^{-1}$ to $T_{1}=950{ }^{\circ} \mathrm{C}$ for one set, and $T_{1}=800{ }^{\circ} \mathrm{C}$ for the other, and kept constant for 10 hours. For the subsequent reducing stage $\left(T_{2}\right)$, for both sets the temperature was brought to $T_{2}=850{ }^{\circ} \mathrm{C}$, again at a rate of $12{ }^{\circ} \mathrm{C} \mathrm{min}{ }^{-1}$; a reducing environment was achieved with a continuous flux of $100 \mathrm{~mL}$ $\min ^{-1}$ (set with a mass flow controller Aalborg DFC26) of CO (compressed carbon monoxide tank, Advanced Specialty Gases) in the firing chamber. This temperature for the reducing stage, $T_{2}=850{ }^{\circ} \mathrm{C}$, is the same as that used in our previous replication study in order to facilitate evaluation of the temperature and duration of the two oxidative stages. In the previous study each of the three stages were held for only 30 minutes, and it was found that the temperature of the final oxidizing stage $\left(T_{3}\right)$ needed to be less than $800{ }^{\circ} \mathrm{C}$ to prevent the re-oxidation of black $\mathrm{Fe}^{2+}$ to red $\mathrm{Fe}^{3+}$. In the current study, the sherds were reoxidized at lower temperatures and for longer times: $T_{3}=600{ }^{\circ} \mathrm{C}$ and $T_{3}=700{ }^{\circ} \mathrm{C}$, each for 10 hours.

Cross-sections/thin sections. Samples measuring $5 \mathrm{~mm} \times 5$ $\mathrm{mm}$ were cut from the replicates with a diamond saw (Buehler). Samples of gloss from the ancient sample (EXT and INT) were removed with a scalpel, resulting in fragments about $180 \mu \mathrm{m} \times$ $50 \mu \mathrm{m}$. All the samples were embedded in resin (SpeciFix epoxy resin added with SpeciFix-20 curing agent, $5: 1$ ratio) and cured for 24 hours at room temperature. After drying the embedded samples were polished with SiC paper (Buehler grinding paper P400, P600, P1200, P4000) to obtain thin sections of about $50 \mu \mathrm{m}$.

\section{Analytical techniques}

$\mathrm{X}$-ray nano- and micro-spectroscopy. Two types of X-ray micro-spectroscopies were performed: X-ray fluorescence 
spectroscopy (XRF) and full-field transmission X-ray microspectroscopy (FF-XANES) across the Fe K edge.

$\mathrm{XRF}$ data were collected at the hard X-ray microprobe located at beamline 2-3 at the Stanford Synchrotron Radiation Lightource (SSRL). Monochromatic radiation was extracted from the white beam bend magnet spectrum by two parallel $\mathrm{Si}(111)$ crystals, and focused to a $2 \mu \mathrm{m}$ spot using a Rh-coated $\mathrm{K}-\mathrm{B}$ mirror pair. For XRF measurements the incident X-rays were monochromatized to $10 \mathrm{keV}$. The data were collected by a single channel solid state detector (Vortex), and digitally processed to obtain the full fluorescence spectrum at every point. The fluorescence maps were analysed with the SMAK 1.10 software package. $^{\mathbf{4 3}}$

FF-XANES data were collected at beamline 6-2c at SSRL in the full-field transmission X-ray microscope (TXM) and at the X-ray micro-spectroscopy beamline ID21 at the European Synchrotron Radiation Facility (ESRF), in full-field configuration, at the Fe K edge (7.1 keV). For the ESRF ID21 full-field instrument, an undulator coupled with a fixed-exit double-crystal monochromator equipped with a $\mathrm{Si}(111)$ crystal allowed selection of the energy of the primary radiation. The beam size was defined using slits and the beam's spatial structures were smoothed using an X-ray decoheror (graphite foil, $125 \mu \mathrm{m}$ thick). Samples were mounted vertically, perpendicular to the beam, and radiographies were acquired with a detection ensemble comprised of a scintillator, magnifying objective and CCD camera. The ESRF configuration resulted in images with a pixel size of 300 $\mathrm{nm}$ and a field of view of $630 \times 720 \mu \mathrm{m}^{2}$; the SSRL configuration resulted in a nominal image resolution of $30 \mathrm{~nm}$ and a field of view of $30 \times 30 \mu^{2}$. At SSRL, three adjacent images were stitched together into a mosaic to extend the field of view. Transmission full-field images were collected from 7.05 to 7.25 keV. Full-field transmission images were compiled and registered to eliminate misalignment and jitter either by Fast Fourier Transform (FFT) or Scale Invariant Feature Transpose (SIFT). ${ }^{44}$ Each pixel of the thus registered and compiled image stack contains a full X-ray absorption (XAS) spectrum. The first 10-20 (pre-edge) points and the last 20-50 (post-edge) points were used to normalize the XAS. The difference between the average of the pre-edge and the post-edge was extracted as the edge jump. Average variation in the pre-edge was calculated for each image-stack pixel, and if the edge jump for that pixel was less than 8 times the pre-edge variation, then that pixel was eliminated from further analysis. This edge-jump filtering mostly removed pixels with low absorbing areas, and even after filtering between $0.3-3 \mathrm{M}$ pixels remained. The XAS spectra of the remaining pixels (edge-jump filtered) were normalized to make the difference between the pre-edge and post-edge equal to unity. The energy at which the normalized XANES spectra crossed 0.5 was extracted for each retained pixel; the thus extracted edge position (edge shift) is directly related to the oxidation state of Fe. Further details of the FF-XANES experimental setup at SSRL and ESRF and data processing are given elsewhere. ${ }^{45}$

The large number of XANES spectra available opens the possibility of applying statistically driven unsupervised data analytics techniques, and our experience with such an approach will be reported in a separate publication. In here, we will stay with the traditional supervised and interactive curation approach. In the supervised approach, we sampled spectra from several interesting regions of the samples and compared them with Fe mineralogical reference spectra maintained by the Advance Lightsource, Lawrence Berkeley National Lab. ${ }^{46}$

\section{Results}

\section{Berlin painter sherd}

Fig. $2 \mathrm{a}$ and $\mathrm{b}$, and $3 \mathrm{a}$ and $\mathrm{b}$, show the optical image and corresponding edge shift map derived from the FF-XANES data collected at ESRF ID21 for the EXT and INT cross-sections of the ancient sherd, respectively. The edge shift maps of both crosssections show a thin reduced top layer over a more oxidized base; not an unexpected result.

For the sample from the exterior (EXT) surface, in which the observed stratigraphy is black gloss over red gloss over red body, the average spectra of single pixel XANES acquired at beamline ID-21 in each of these three regions are shown in Fig. 2d (solid lines); reference spectra of the Fe minerals that appear to be good matches for each of the regions (hercynite, hematite and maghemite) from the ALS XANES library are shown as dotted lines. The XANES spectrum for every retained pixel was fit as a linear combination of the three identified reference mineral spectra. Fig. 2c shows an RGB map derived from the linear combination least square (LCLS) fit, with pure red assigned to hematite (the $\alpha$-phase of $\mathrm{Fe}_{2} \mathrm{O}_{3}$ ), pure green assigned to hercynite $\left(\mathrm{FeAl}_{2} \mathrm{O}_{4}\right)$ and pure blue assigned to maghemite (the $\gamma$-phase of $\mathrm{Fe}_{2} \mathrm{O}_{3}$ ). The elimination of any of the three reference spectra from the LCLS fitting significantly degrades the fit for a substantial number of pixels with large edge jump (and hence low noise). Interestingly, the addition of other Fe minerals, notably magnetite (which was detected in this sample by Raman spectroscopy), does not significantly improve the LCLS fit for pixels with a large edge jump. Furthermore, principal component analysis (not shown here) also indicates the presence of at least three components and thus the choice of the three references appears reasonable based on the data. The phase map shows that the black gloss is composed of hercynite, the red gloss is composed of hematite, and the body is a mixture of maghemite and hematite.

The results of FF-XANES measurements performed on the sample taken from the interior of the vessel (INT), in which the observed stratigraphy is black gloss over red body, are shown in Fig. 3. As for the EXT sample, the average spectra of single pixel XANES in each region is shown in Fig. 3d (solid lines), with spectra of the Fe minerals found to be good matches (hercynite and maghemite) shown as dotted lines. An RGB phase map showing the mineralogical distribution of hercynite, hematite, and maghemite, derived, as above, from a LCLS fit to reference spectra, is shown in Fig. 3c. This phase map brings out extra detail not immediately apparent from the single pixel XANES spectra, notably that while the black gloss is composed of hercynite the body is a mixture of maghemite and hematite. In addition, although not visible in the optical image, the phase map shows two small layers (each about $5 \mu \mathrm{m}$ thick) at the 

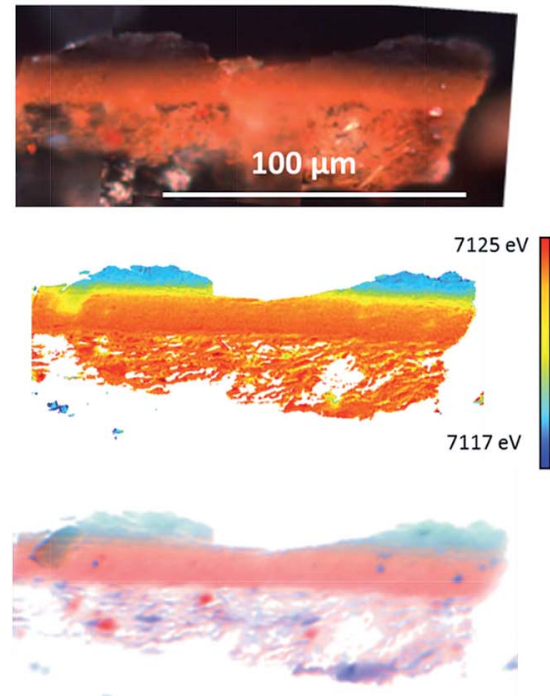

b)

c)

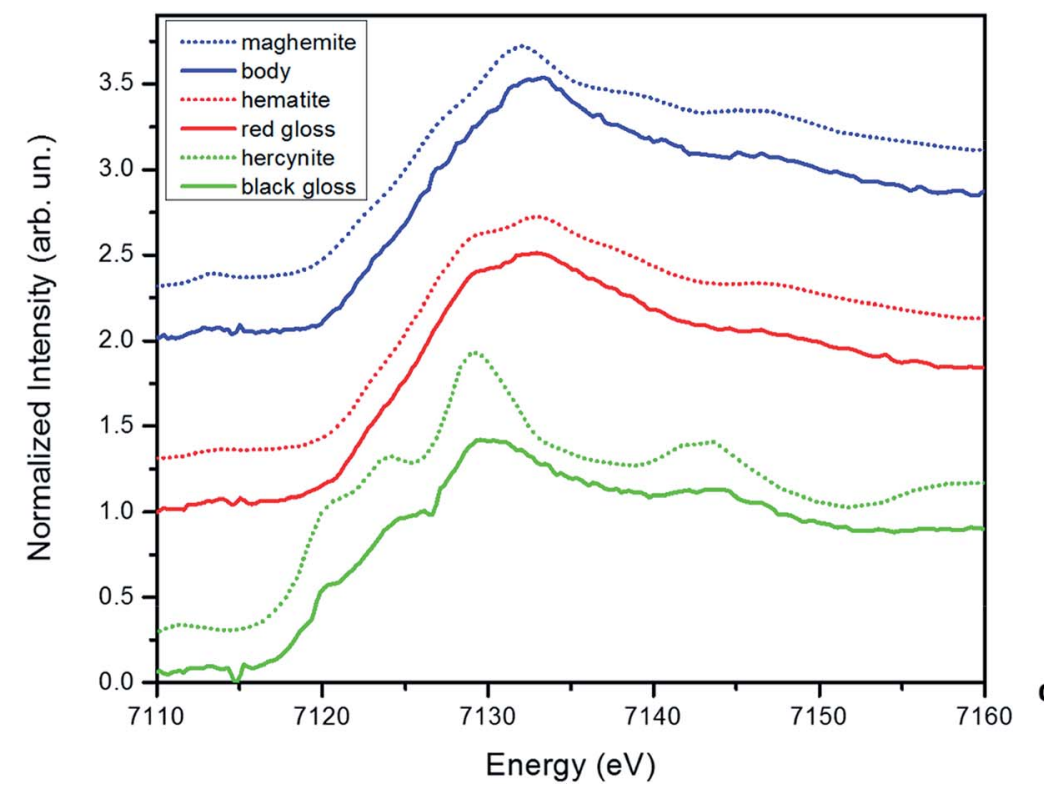

Fig. 2 (a) Optical photomicrograph of EXT. (b) Fe-edge position map with colour-energy scale. (c) RGB phase map generated by the fit of single pixel XANES spectra with iron oxide standards. The map shows that the black gloss is composed of hercynite (green), the red gloss of hematite (red) and the body of a mixture of maghemite (blue) and hematite (red). (d) Averaged spectra of black gloss (solid green line), red gloss (solid red line) and body (solid blue line) collected at ESRF, BL ID-21 and standard spectra of hercynite (dotted green line), hematite (dotted red line) and maghemite (dotted blue line).

interface between the black gloss and the body: a layer of hematite over a layer of maghemite.

To better understand the slip stratigraphy for these two samples, each of which show, to different extents, a reduced gloss layer over oxidized gloss layer(s), we need to compare the high resolution FF-XANES measurements from beamline 6-2c with optical and XRF measurements. Fig. 4a shows the optical image of the cross-section from the EXT sample, in which the black gloss, red gloss and body are clearly visible. XRF maps (Fig. 4b) show that the body contains more $\mathrm{Ca}$, present in large
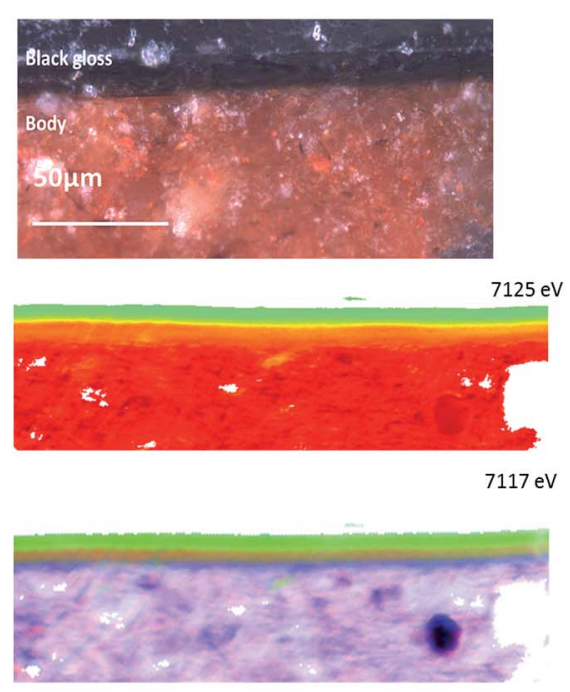

a)

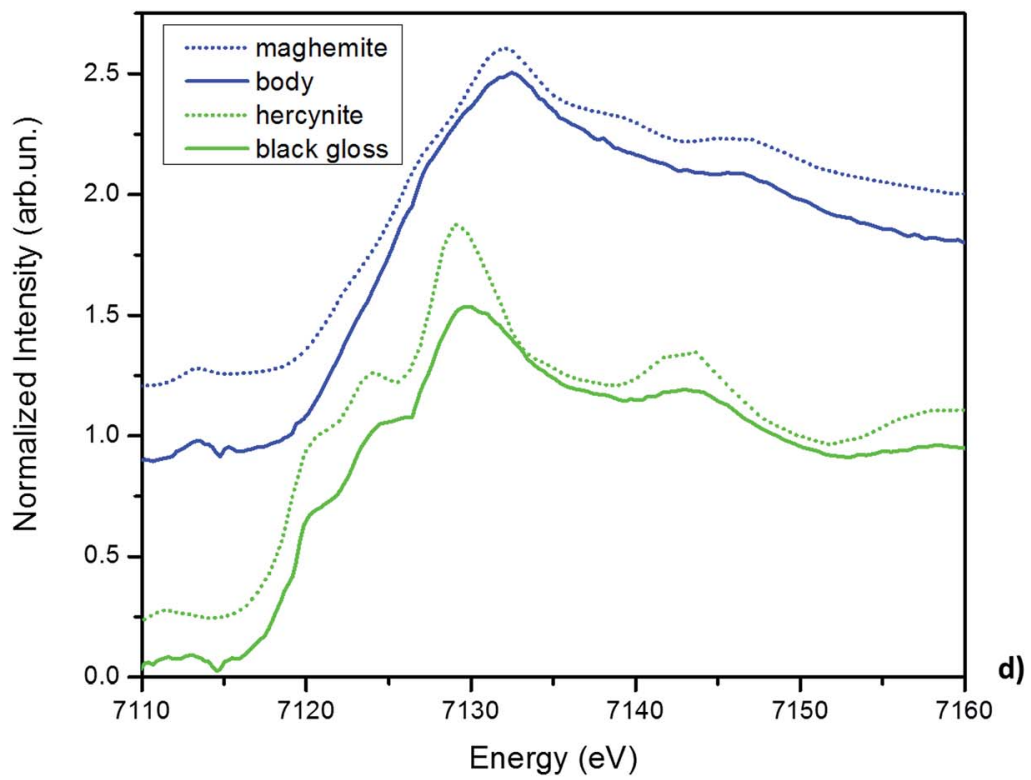

Fig. 3 (a) Optical photomicrograph of INT. (b) Fe-edge position map with colour-energy scale. (c) RGB phase map generated by the fit of single pixel XANES spectra with iron oxide standards. The map shows that the black gloss is composed of hercynite and the body of a mixture of maghemite and hematite. A small layer (about $5 \mu \mathrm{m}$ ) of hematite is observed at the interface between the black gloss and the body. (d) Averaged spectra of the black gloss (solid green line) and body (solid blue line) collected at ESRF, BL ID-21 and standard spectra of hercyinite (dotted green line) and maghemite (dotted blue line). 
crystallites or clusters, than either of the gloss layers; otherwise the bulk chemistry of two slips and the body are very similar (further details on the composition is given in our previous study $\left.^{26}\right)$. Because the overall chemistry of both slips and the body are very similar, X-ray absorbance of a uniformly thin cross-section, below the Fe $\mathrm{K}$ edge, is a good measure of the local density and inversely related to the porosity. Fig. 4c shows the absorbance of the EXT cross-section measured at beamline 6-2c at $7080 \mathrm{eV}$ (below the absorption edge of Fe), which clearly shows that the body is significantly more porous than the two gloss layers, and both gloss layers appear to have very comparable density. However, notably, the bottom of the red gloss (and very topmost surface of the black gloss) show slightly increased density. Fig. 4d shows the Fe K-edge-shift map for the cross-section, which indicates that, as expected, the iron in the black gloss is reduced, while the iron in the red gloss and body are both oxidized, with patches of even higher oxidation throughout the body. Furthermore, although not shown, the very top surface (extending down no more than 1-2 $\mu \mathrm{m}$ ) of the black gloss layer, appears to be further reduced than the bulk of the black gloss layer. Fig. 4e shows the phase map for the sample (generated using the same LCLS method applied to the lower resolution data as shown in Fig. 2 and 3). This high resolution phase map clearly shows the distribution of hercynite (green), hematite (red), and maghemite (blue) in the EXT cross-section: the heart of the black gloss layer is composed mostly of hercynite, the red gloss layer is composed mostly of hematite, and the broad transition region between the two containing strands of maghemite in a hematite or hercynite matrix. The body, on the other hand, shows a mixture of hematite and maghemite, with more dense grains composed of hematite. The dense and granular nature of hematite regions in the body is even clearer here than in the phase maps shown in Fig. $2 \mathrm{c}$ and $3 \mathrm{c}$.

\section{Replicate sherds}

Replication studies were conducted in order to help decipher the firing sequences and other production processes necessary to create a ceramic vessel with the morphological and mineralogical distribution found in the Berlin Painter's sherd, described above. A series of replicates were produced to explore the processing envelope achievable in a large wood-fired kiln that would enable the production of (a) a dense red gloss layer (such as that observed under the black gloss layer on the sample EXT) and (b) a high quality black gloss layer over a fully oxidized red body (such as that observed on the sample INT). In Table 1 the firing conditions of the replicates thus produced are reported, together with the main mineralogical phases detected in the body and the slip by Raman spectroscopy and by XANES. In the last column the denomination of the slip and the optical image of the cross-sections are also reported.

\section{Formation of red gloss}

In our previous study ${ }^{26}$ it was found that firing at $T_{1}=1000{ }^{\circ} \mathrm{C}$ for only 30 minutes could induce hematite precipitation in the slip to form red gloss. However, this temperature is not believed to be realistically achievable in an ancient wood-fired kiln, ${ }^{47}$ nor would such a short firing have been employed. Thus, in the present study we fired the replicate sherds at lower temperatures and for longer times. Painted replicate sherds were fired at $T_{1}=950{ }^{\circ} \mathrm{C}$ under oxidizing conditions for 10 hours, then reduced at $T_{2}=850{ }^{\circ} \mathrm{C}$ for 30 minutes. As seen in Table 1, row 1 , under these conditions the slip layer is transformed into a
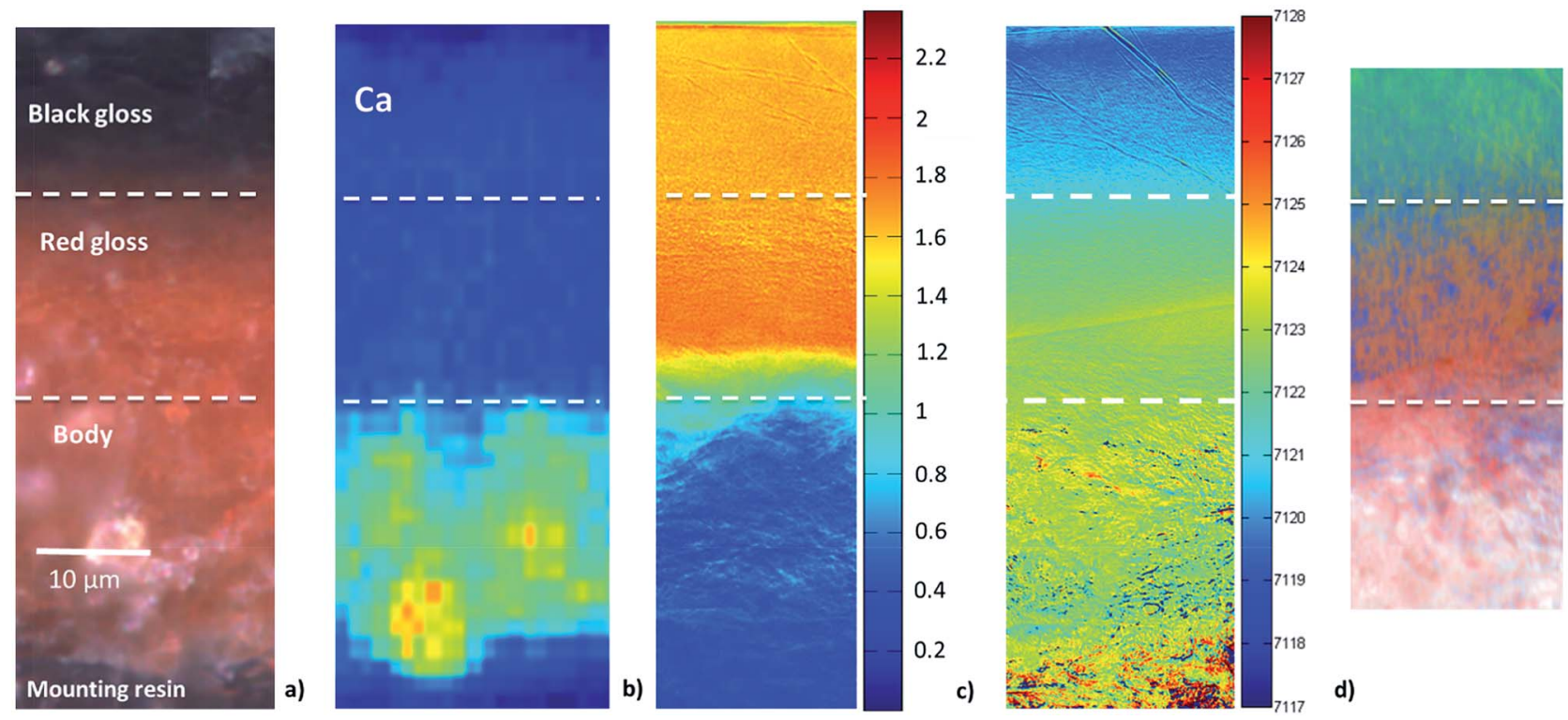

e)

Fig. 4 (a) Cross-section of sample EXT. (b) Ca XRF map. (c) X-ray absorption image at 7080 eV showing different porosity in the two glosses compared to the body. (d) Fe-edge position map. (e) RGB phase map generated by the fit of single pixel XANES spectra with iron oxide standards. The phase map shows that the black gloss is composed of hercynite (green), the red gloss of hematite (red) and the body of a mixture of maghemite (blue) and hematite. 
Table 1 Firing conditions of replicates with relative colour of gloss and body and iron species detected

\begin{tabular}{llllll}
\hline Row & $T_{1}\left({ }^{\circ} \mathrm{C}\right)$ & $T_{2}\left({ }^{\circ} \mathrm{C}\right)$ & $T_{3}\left({ }^{\circ} \mathrm{C}\right)$ & Colour & Main Fe species
\end{tabular}

1950 for $10 \mathrm{~h} \quad 850$ for $30^{\prime} \quad$ NA $\quad \begin{array}{lll}\text { Gloss } & \text { Red } & \text { Hematite (Raman, XANES) } \\ \text { Body } & \text { Black } & \text { Hercynite (XANES), Magnetite (Raman) }\end{array}$

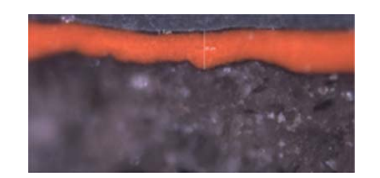

Coral red type II

2950 for $10 \mathrm{~h} \quad 850$ for $30^{\prime} \quad 800$ for $30^{\prime} \quad \begin{array}{lll}\text { Gloss } \\ \text { Body }\end{array} \quad \begin{aligned} & \text { Red } \\ & \text { Red }\end{aligned} \quad \begin{aligned} & \text { Hematite (Raman, XANES) } \\ & \text { Hematite (Raman), Hematite } \\ & \text { + Maghemite (XANES) }\end{aligned}$

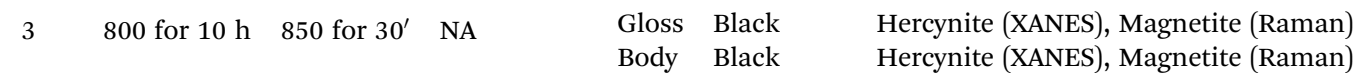
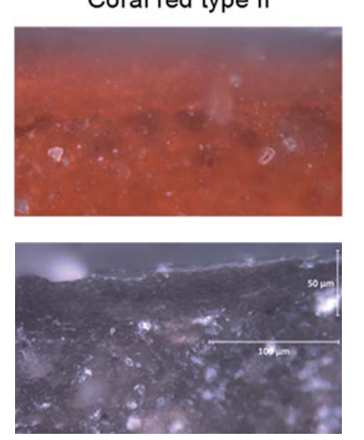

Successful firing

Gloss Black Hercynite (XANES), Magnetite (Raman) Body Red Hematite (Raman), Hematite + Maghemite (XANES)

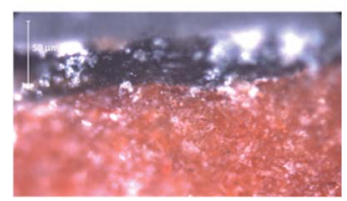

Succesful firing

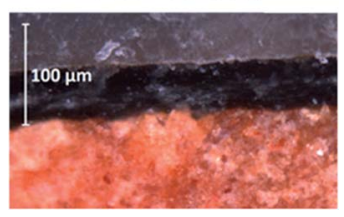

Misfire T3

Hematite (Raman), Hematite +

Maghemite (XANES) vitrified red gloss, with only a thin layer of black formed at the surface during the reduction step (the body, being porous, is completed reduced by the CO). This same replicate was subsequently re-oxidized at $T_{3}=800{ }^{\circ} \mathrm{C}$ for 30 minutes (Table 1 , row 2) and now the body and the slip both appear red. These data show that a longer firing at a lower temperature can produce the same results as a shorter firing at a higher temperature.

\section{Formation of black gloss}

In our previous study ${ }^{26}$ black gloss was obtained by firing replicate sherds under oxidizing conditions at either $T_{1}=$ $800{ }^{\circ} \mathrm{C}$ or $900{ }^{\circ} \mathrm{C}$ for 30 minutes (followed by a reduction firing at $T_{2}=850^{\circ} \mathrm{C}$ for 30 minutes). Analogous to that observed for the formation of red gloss described above, firing at a lower temperature $\left(T_{1}=800^{\circ} \mathrm{C}\right)$ for a longer time (10 hours) produced a homogenous, smooth black slip (Table 1, row 3 ) similar to that produced by firing at $T_{1}=900{ }^{\circ} \mathrm{C}$ for 30 minutes. By contrast, firing at $T_{1}=900{ }^{\circ} \mathrm{C}$ for 10 hours (image not shown) was sufficient to induce local precipitation of hematite in some areas of the slip, resulting in what is often termed as "misfired": areas intended to be black have, in fact, turned red. Therefore, to produce black gloss using longer firing times, lower temperatures, with $T_{1}<900{ }^{\circ} \mathrm{C}$, should be used for the first oxidizing stage in the firing sequence.

\section{Re-oxidation of the body}

Ideally, in the final, re-oxidation step, the reduced iron in the porous clay body is reverted to an oxidized form, while the iron species in the vitrified slips are unaffected, remaining either red (oxidized, formed at high temperatures in the initial oxidizing stage) or black (reduced, formed at lower temperatures in the initial oxidizing stage). To test whether, like that observed in the 
initial oxidizing stage, firing at high temperatures for short times yields similar results to firing at lower temperatures for longer times, replicates fired at $T_{1}=800{ }^{\circ} \mathrm{C}$ (10 hours) were reoxidized at $T_{3}=800{ }^{\circ} \mathrm{C}$ for 30 minutes (Table 1, row 4) and at $T_{3}=600{ }^{\circ} \mathrm{C}$ and $700{ }^{\circ} \mathrm{C}$ for 10 hours (Table 1 , rows 5 and 6 , respectively).

For the two samples subjected to longer firing times for the re-oxidation step (10 hours), at $T_{3}=600{ }^{\circ} \mathrm{C}$ the black gloss was unaffected, while at $T_{3}=700{ }^{\circ} \mathrm{C}$ the black gloss was re-oxidized to red. By contrast, when a short firing time (30 minutes) was used (see Table 1, row 4), even the higher temperature of $T_{3}=$ $800{ }^{\circ} \mathrm{C}$ did not induce re-oxidation of the black gloss; the gloss remained black. Therefore, we conclude that to produce black gloss using long firing times, the final re-oxidation step should be fired at temperatures $T_{3}<700{ }^{\circ} \mathrm{C}$, although higher temperatures can be tolerated for shorter times.

XANES spectroscopy was used to characterize the mineral phases in the slips and the body of the replicate sherds. Phase maps generated by the LCLS fitting of the XANES spectra of the replicates with the ALS standards are shown in Fig. 5. The same colour coding used for the ancient sample was adopted here: pure red for hematite $\left(\alpha-\mathrm{Fe}_{2} \mathrm{O}_{3}\right)$, pure green for hercynite $\left(\mathrm{FeAl}_{2} \mathrm{O}_{4}\right)$, and pure blue for maghemite $\left(\gamma-\mathrm{Fe}_{2} \mathrm{O}_{3}\right)$. Fig. $5 \mathrm{~b}$ shows the phase map of the sample produced at $T_{1}=950{ }^{\circ} \mathrm{C}$ and $T_{3}=800{ }^{\circ} \mathrm{C}$ (Table 1, row 2), in which a layer of red gloss is formed over a red body. The red gloss is seen to be mostly
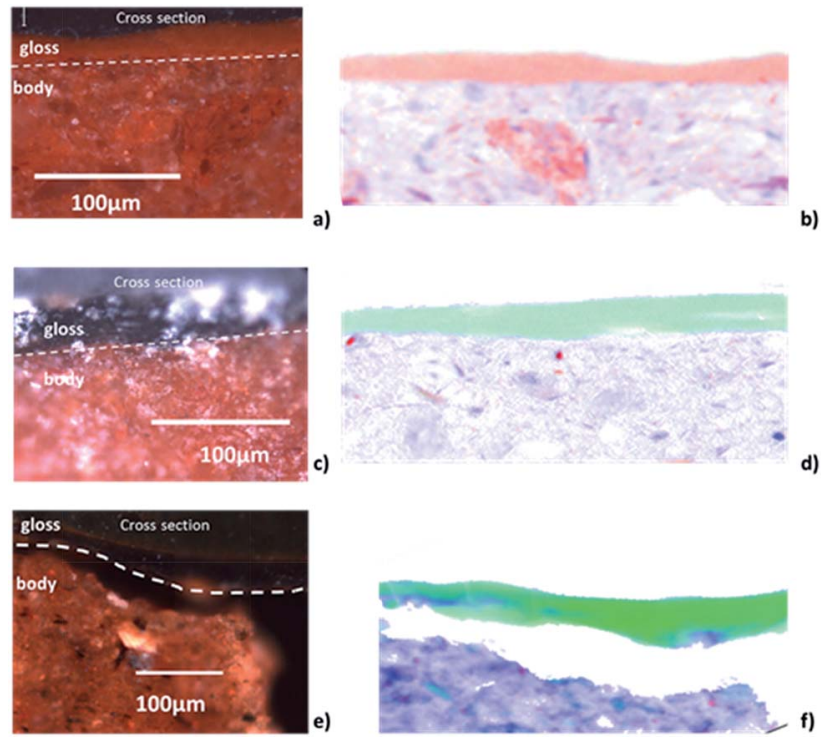

Fig. 5 Cross-sections of replicates produced at different temperatures and duration of the firing steps. Relative RGB maps (red $=$ hematite, green $=$ hercynite, blue = maghemite) generated by LCLS fit with standards. (a) Cross-section of replicate sample fired at $T_{1}=$ $950{ }^{\circ} \mathrm{C}(10 \mathrm{~h}), T_{2}=850^{\circ} \mathrm{C}\left(30^{\prime}\right), T_{3}=800^{\circ} \mathrm{C}\left(30^{\prime}\right)$ showing red gloss over red body. (b) RGB phase map of replicate sample in (a). (c) Crosssection of replicate sample fired at $T_{1}=800{ }^{\circ} \mathrm{C}(10 \mathrm{~h}), T_{2}=850^{\circ} \mathrm{C}$ $\left(30^{\prime}\right), T_{3}=800^{\circ} \mathrm{C}\left(30^{\prime}\right)$ showing black gloss over red body. (d) RGB phase map of replicate sample in (c). (e) Cross-section of replicate sample fired at $T_{1}=800^{\circ} \mathrm{C}(10 \mathrm{~h}), T_{2}=850^{\circ} \mathrm{C}\left(30^{\prime}\right), T_{3}=600^{\circ} \mathrm{C}(10 \mathrm{~h})$ showing black gloss over red body. (f) RGB phase map of replicate sample in (e) (gloss detached from the body). composed of hematite, but a very thin layer of maghemite is observed at the top surface; the body is a mixture of maghemite and hematite. The phase maps of the two samples fired at a lower temperature, $T_{1}=800{ }^{\circ} \mathrm{C}$, and then re-oxidized at $T_{3}=800$ ${ }^{\circ} \mathrm{C}$ for 30 minutes and $T_{3}=600{ }^{\circ} \mathrm{C}$ for 10 hours (Table 1, rows 4 and 5, and Fig. $5 \mathrm{~d}$ and $\mathrm{f}$, respectively), show the same mineralogy: the black slip is composed of hercynite and the body is a mixture of predominantly maghemite with hematite also present, and the topmost layer slightly re-oxidized compared to the bulk. Again, we have evidence that, like that observed in the first oxidizing step, two different conditions of re-oxidation high temperature/short time and lower temperature/longer time - lead to the same result: formation of maghemite, the $\gamma$ phase of $\mathrm{Fe}_{2} \mathrm{O}_{3}$, together with hematite, the $\alpha$-phase of $\mathrm{Fe}_{2} \mathrm{O}_{3}$, in the body. It is worth noting that because of the lower sensitivity of Raman spectroscopy to maghemite its presence in the body only became evident with the XANES studies, which indicate it is, in fact, the major phase present.

\section{Discussion}

\section{Replicate sherds}

A distinct Fe mineralogy is associated with a successful (i.e., the production of black gloss) three-step firing: Fe in the black gloss is predominantly present as hercynite, whereas Fe in the red body is predominantly found as maghemite, with hematite also present. From our replication studies, for long firing times the high temperature boundary for the successful production of black gloss on a red body in a three step (oxidizing-reducingoxidizing) firing sequence was found to be $T_{1}<900{ }^{\circ} \mathrm{C}$ for the first (oxidizing) step and $T_{3}<700{ }^{\circ} \mathrm{C}$ for the third (re-oxidizing) step (with the $T_{2}$ upper boundary intermediate between $T_{1}$ and $T_{3}$ ). The boundaries move to lower temperatures as annealing times are increased. Significant infractions of this boundary, in either of the two oxidizing steps, result in reddening of the intended black gloss and leads to the production of red gloss.

We have not fully explored the low temperature boundary, as that is very heavily dominated by kinetics, although our measurements suggest it is below $700{ }^{\circ} \mathrm{C}$. Significant transgression of the high temperature boundary during the reduction phase can produce metallic $\mathrm{Fe}$ or even wüstite, $\mathrm{Fe}_{1-x} \mathrm{O}$, but these phases are not common in Greek pottery ${ }^{5,37,42}$ as it is very difficult to achieve elevated temperatures under very reducing conditions in a wood-fired kiln, and thus we have not explored this regime in the replication studies.

Our replication studies show two pathways for forming red (oxidized) gloss in a three-stage firing sequence, but these two pathways result in very different microstructure and mineralogy. If the matrix of the slip is sufficiently porous after the second, reducing, stage, oxygen can easily permeate it and reoxidize the gloss. This type of red gloss is formed through the process of maghemitization of the $\mathrm{Fe}^{2+}$ (ferrous) spinels (hercynite and magnetite) ${ }^{21}$ and a significant fraction of $\mathrm{Fe}^{3+}$ (ferric) ions thus produced will be in the maghemite phase. Higher permeability of the red gloss layer than that of the black gloss layer is achieved through a change in the bulk clay chemistry (e.g., large calcite content). One of the two identified types of 
Coral Red is consistent with being produced by this mechanism. ${ }^{9}$ The other pathway for forming red slips in a three-stage firing is to make the matrix sufficiently impermeable in the first (or before the first) oxidative step so that it cannot be reduced in the second stage. This can be achieved by a prolonged annealing at elevated temperatures in which the aluminum-silicate matrix is almost fully vitrified. Distinguishing material characteristics of red gloss produced by this pathway are an even higher degree of vitrification than black gloss and the presence of large amounts of hematite. The second type of Coral Red previously observed, in which red and black gloss layers have similar chemistry, ${ }^{9}$ would have been produced through this mechanism.

Thus, our replication studies have shown that the mineralogy associated with the three-stage firing envelope not only provides "finger-prints" for deciphering the firing sequence from material characterization, it also provides the scientific foundation for understanding nanoscale processes occurring at various stages of production of black and red glosses.

\section{Berlin Painter sherd}

The red gloss found on the EXT sample from the Berlin Painter sherd was found to be slightly more dense than the overlying black gloss (see Fig. 4c), and hence belongs to the second category of red gloss described above. Furthermore, since the bulk chemistry of the red gloss is essentially indistinguishable from that of the black gloss (see Fig. 4b), the higher density must have been achieved by firing at a higher temperature. That a high firing temperature was employed is confirmed by the presence of a large amount of hematite in the heart of the red gloss layer, as shown in Fig. 4e. However, the high temperature firing needed to vitrify the red gloss would have also vitrified the black gloss (with identical chemistry), if it was present. Therefore we conclude that the decorative black gloss layer must have been applied after the high temperature annealing that produced the red gloss, and fired in a second, separate threestage firing at lower temperature(s).

This seemingly difficult formation of a layer of black gloss on top of a layer of red gloss is made possible by the different behaviour of iron containing aluminum-silicates under reducing or oxidizing conditions. The viscosity of iron containing silicates has been found to decrease under reducing conditions, and the glass transition temperature of these compounds is lower in presence of $\mathrm{Fe}^{2+}$ compared to $\mathrm{Fe}^{3+} \cdot{ }^{48}$ Because the melting point of the top black layer is lower than that of the red gloss layer beneath it, it is possible to vitrify the top slip without affecting the one underneath, while selectively reducing the upmost layer.

The presence of a thin, dense, oxidized layer under the black gloss in the INT sample (visible in the mineralogical mapping (see Fig. 3c) but not optically), also supports the hypothesis that a second application of refined clay was applied after the first oxidative step. As noted above, this layer is actually comprised of three layers: a thin maghemite layer at the interface with the body, over which is found an equally thin hematite layer, which lies directly under the main black gloss (hercynite) layer. The most likely scenario for creation of these thin layers, based on the observed mineralogical distribution is an initial high temperature firing followed by an application of the gloss layer and subsequent lower temperature oxidizing-reducingoxidizing firing. Specifically, the initial high temperature firing (annealing) used to create the thick red gloss layer on the exterior surface as described above also results in the formation of a thin hematite layer at the outer surface of the (unpainted) body clay on the interior of the vessel. This is followed by subsequent painting of the decorative motif (areas intended to be black) on both the interior and exterior surfaces, which is then subjected to a second (three-stage) firing of the vessel. Because of the high density of the hematite layer thus produced, only the very bottom surface (in contact with the porous body) is reduced during the (reducing) stage of this second firing, which in turn is transformed into maghemite in the third re-oxidizing step.

The presence of significant amounts of hematite in the body needs further investigation to be fully understood. The maghemitization of ferrous spinels depends strongly on many different parameters: temperature ${ }^{49}$ size and crystal face of the particles, ${ }^{\mathbf{1 9 , 2 0 , 2 3}}$ and chemistry of the clay. ${ }^{50}$ A high resolution $3 \mathrm{D}$ distribution of the hematite, maghemite and the open pores for a large region of the body would allow a better understanding of the re-oxidation process and the duration of the last firing step but such measurement is beyond the scope of the current work.

\section{Conclusions}

The comparison of the mineralogical phases in the sample attributed to the Berlin Painter with those formed under known conditions in the replication study confirms the results of our previously reported Raman study ${ }^{\mathbf{2 6}}$ and further refines our understanding of the firing protocol employed for the production of the ancient sample JPGM 81.AE.206.d.2005. In particular, the conditions required for the formation of the two $\mathrm{Fe}^{3+}$ phases, $\alpha-\mathrm{Fe}_{2} \mathrm{O}_{3}$ (hematite) and $\gamma-\mathrm{Fe}_{2} \mathrm{O}_{3}$ (maghemite), are very different: temperatures as high as $950{ }^{\circ} \mathrm{C}$ are necessary to induce hematite formation (in either the first or third oxidizing stage of firing) while, depending on the duration of the last reoxidation stage, temperatures lower than $800{ }^{\circ} \mathrm{C}$ are required to form maghemite. This means that, to achieve the gloss architecture observed on the surface of the Berlin Painter vessel, in which a homogeneous red glossy layer is found underneath the black gloss on the exterior surface, but only a very thin layer of red gloss is found under the black gloss on the interior surface, two separate applications of slip and two separate firings must have been performed. First, a homogeneous application of slip was applied to the exterior surface, and the vessel was fired at high temperature to form hematite in the vitrified slip. The decorations on the vessel (areas intended to be black) were subsequently painted with a second application of slip followed by a three-stage firing, in which hercynite and magnetite were formed in the black gloss, and hematite and maghemite were formed in the body.

It is important to note that the key parameter to obtain this layered structure is the large degree of vitrification of the two 
slips. In fact, after the precipitation of hematite in the red gloss layer, the densification of the matrix prevents the reduction of iron from occurring during the reducing step. However, it is necessary to undergo a reducing step to form the black layer on top, and thus the temperature used during the firing of the top black layer had to be sufficiently high to guarantee the vitrification of the black gloss, but significantly lower than $950{ }^{\circ} \mathrm{C}$ to avoid the precipitation of hematite.

The presence of a stratified structure, such as that observed in the Berlin Painter vessel, has not been encountered very often in our studies. Thus, further investigations are necessary to determine what factors (i.e., technological, aesthetic or economic) might have motivated - or enabled - the potter to undertake such a complex firing scheme, and how widespread this practice was among contemporary painters and potters. Nevertheless, this study presents yet more evidence supporting the hypothesis that the firing practices of the Kerameikos artists were more complex and less standardized that previously thought, involving multiple firing $\mathbf{s}^{\mathbf{1 0}}$ and the sequential application of slips.

\section{Acknowledgements}

This material is based upon work supported by the National Science Foundation under Grant no.104808. Some of the measurements reported in here were performed on beamline 62c at the Stanford Synchrotron Radiation Lightsource, a Directorate of SLAC National Accelerator Laboratory and an Office of Science User Facility operated for the U.S. Department of Energy Office of Science by Stanford University. Other measurements were performed on beamline ID21 at the European Synchrotron Radiation Facility (ESRF), Grenoble, France, as part of proposal HG27. We would like to thank B. Hesse and B. Fayard (ESRF) and S. Webb, C. Krest, and J. Hayter (SSRL)for their assistance. This work was partially supported by the NEXT labex through the PhD student mobility program.

\section{Notes and references}

1 C. F. Binns and A. D. Frazer, Am. J. Archaeol., 1929, 1-9.

2 J. V. Noble, Am. J. Archaeol., 1960, 64, 307-313.

3 M. Farnsworth and H. Wisely, Am. J. Archaeol., 1958, 62, 165173.

4 G. M. A. Richter, The craft of Athenian pottery: an investigation of the technique of black-figured and red-figured Athenian vases, New Haven. 1923.

5 A. Kostikas, A. Simopoulos and N. H. Gangas, J. Phys. Colloq., 1974, 35, C1-C107-C101-115, DOI: 10.1051/ jphyscol:1974133.

6 M. Walton, E. Doehne, K. Trentelman, G. Chiari, J. Maish and A. Buxbaum, in Papers on Special Techniques in Athenian Vases, ed. K. Lapatin and J. Paul Getty Museum, 2008, pp. 95-104.

7 F. Ospitali, T. Sabetta, F. Tullini, M. C. Nannetti and G. Di Lonardo, J. Raman Spectrosc., 2005, 36, 18-23, DOI: 10.1002/jrs.1259.
8 Y. Maniatis, E. Aloupi and A. D. Stalios, Archaeometry, 1993, 35, 23-34, DOI: 10.1111/j.1475-4754.1993.tb01021.x.

9 M. Walton, K. Trentelman, E. Doehne, G. Chiari, J. Maish and A. Buxbaum, Archaeometry, 2009, 51, 383-396, DOI: 10.1111/j.1475-4754.2008.00413.x.

10 M. S. Walton, K. Trentelman, M. Cummings, G. Poretti, J. Maish, D. Saunders, B. Foran, M. Brodie, A. Mehta and P. Vandiver, J. Am. Ceram. Soc., 2013, 96, 2031-2035, DOI: 10.1111/jace.12395.

11 M. S. Walton, M. Svoboda, A. Mehta, S. Webb and K. Trentelman, Journal of Archaeological Science, 2010, 37, 936-940, DOI: 10.1016/j.jas.2009.11.026.

12 M. Walton and K. Trentelman, Materials Research Society Symposium: Materials Issues in Art and Archaeology VIII, 2007.

13 P. Artal-Isbrand and P. Klausmeyer, Stud. Conserv., 2013, 58, 338-359, DOI: 10.1179/2047058412y.0000000077.

14 L. Kahn and J. Wissinger, in The Colors of Clay: Special Techniques in Athenian Vases, J Paul Getty Museum, Los Angeles. 2006.

15 W. D. Kingery, Archeomaterials, 1991, 5, 47-54.

16 Y. Maniatis, A. Simopoulos, A. Kostikas and V. Perdikatsis, J. Am. Ceram. Soc., 1983, 66, 773-781.

17 L. Luhl, B. Hesse, I. Mantouvalou, M. Wilke, S. Mahlkow, E. Aloupi-Siotis and B. Kanngiesser, Anal. Chem., 2014, 86, 6924-6930, DOI: 10.1021/ac500990k.

18 F. Meirer, Y. Liu, E. Pouyet, B. Fayard, M. Cotte, C. Sanchez, J. C. Andrews, A. Mehta and P. Sciau, J. Anal. At. Spectrom., 2013, 28, 1870, DOI: 10.1039/c3ja50226k.

19 A. Navrotosky, L. Mazeina and J. Majzlan, Science, 2008, 319, 1635-1638.

20 K. Haneda and A. H. Morrish, J. Phys., 1977, 38, 321-323.

21 D. Dunlop and Ö. Özdemir, Rock Magnetism: Fundamentals and Frontiers, Cambridge University Press, Cambridge. 1997.

22 H. Fischer, J. Luster and A. U. Gehring, GEOPHYS. J. INT., 2007, 169, 909-916.

23 K. F. McCarty, M. Monti, S. Nie, D. A. Siegel, E. Starodub, F. El Gabaly, A. H. McDaniel, A. Shavorskiy, T. Tyliszczak, H. Bluhm, N. C. Bartelt and J. de la Figuera, J. Phys. Chem. C, 2014, 118, 19768-19777.

24 C. Nunes, E. C. Resende, I. R. Guimaraes, A. S. Anastacio and M. C. Guerreiro, Appl. Spectrosc., 2011, 65, 692-697.

25 M. Wilke, F. Farges, P. E. Petit, G. E. Brown, Jr and F. Martin, Am. Mineral., 2001, 86, 714-730.

26 I. Cianchetta, J. Maish, D. Saunders, M. Walton, A. Mehta, B. Foran and K. Trentelman, J. Raman Spectrosc., 2014, just accepted.

27 A. Zoppi, C. Lofrumento, E. M. Castellucci and M. G. Migliorini, Ann. Chim., 2005, 95, 239-246.

28 Y. Leon, C. Lofrumento, A. Zoppi, R. Carles, E. M. Castellucci and P. Sciau, J. Raman Spectrosc., 2010, 41, 1550-1555, DOI: 10.1002/jrs.2678.

29 Y. Leon, P. Sciau, P. Goudeau, N. Tamura, S. Webb and A. Mehta, Appl. Phys. A: Mater. Sci. Process., 2010, 99, 419425, DOI: 10.1007/s00339-010-5628-x.

30 P. Sciau, Y. Leon, P. Goudeau, S. C. Fakra, S. Webb and A. Mehta, J. Anal. At. Spectrom., 2011, 26, 969, DOI: 10.1039/c0ja00212g. 
31 E. Aquilia, G. Barbera, G. Barone, V. Crupi, F. Longo, D. Majolino, P. Mazzoleni and V. Venuti, X-Ray Spectrom., 2013, 42, 38-44, DOI: 10.1002/xrs.2432.

32 V. S. F. Muralha, T. Rehren and R. J. H. Clark, J. Raman Spectrosc., 2011, 42, 2077-2084, DOI: 10.1002/jrs.2961.

$33 \mathrm{H}$. Edwards and J. M. Chalmers, Raman Spectroscopy in Archaeology and Art History, RCS, 2005.

34 T. Broekmans, A. Adriaens and E. Pantos, Appl. Phys. A: Mater. Sci. Process., 2007, 90, 35-42, DOI: 10.1007/s00339007-4227-y.

35 E. Gliozzo, I. W. Kirkman, E. Pantos and I. Memmi Turbanti, Archeometry, 2004, 46, 227-246.

36 .R. project.

37 F. Bardelli, G. Barone, V. Crupi, F. Longo, G. Maisano, D. Majolino, P. Mazzoleni and V. Venuti, J. Synchrotron Radiat., 2012, 19, 782-788, DOI: 10.1107/ S0909049512023990.

38 D. Barilaro, V. Crupi, D. Majolino, V. Venuti, G. Barone, F. D'Acapito, F. Bardelli and F. Giannici, J. Appl. Phys., 2007, 101, 064909, DOI: 10.1063/1.2537908.

39 A. O. Shepard, Ceramics for the Archaeologist, Carnegie Institution of Washington, Washington D.C., 1956.
40 G. M. Ingo, E. Angelini, G. Bultrini, T. De Caro, L. Pandolfi and A. Mezzi, Surf. Interface Anal., 2002, 34, 328-336, DOI: 10.1002/sia.1311.

41 G. M. Ingo, G. Bultrini, T. de Caro and C. Del Vais, Surf. Interface Anal., 2000, 30, 101-105.

42 C. C. Tang, E. J. MacLean, M. A. Roberts, D. T. Clarke, E. Pantos and A. J. N. W. Prag, J. Archaeol. Sci., 2001, 28, 1015-1024, DOI: 10.1006/jasc.2000.0608.

43 S. Webb, 2014.

44 P. Paleo, E. Pouyet and J. Kieffer, J. Synchrotron Radiat., 2014, 21, 456-461.

45 B. Fayard, E. Pouyet, G. Berruyer, D. Bugnazet, C. Cornu, M. Cotte and A. Sole, J. Phys.: Conf. Ser., 2013, 25, 1-4.

46 XAS database of Fe compounds, Advanced Light Source (ALS), Lawrence Berkeley National Laboratory, California, Website: http://xraysweb.lbl.gov/uxas/Databases/Overview.htm.

47 N. Cuomo di Caprio, Ceramiche in Archeologia 2, L'Erma di Bretschneider., 2007.

48 L. C. Klein, B. V. Fasano and J. M. Wu, Lunar and Planetary Science Conference, 13th, Houston, TX.

49 I. I. Diakonov, Eur. J. Mineral., 1998, 10, 17-29.

50 O. Helgason, H. P. Gunnlaugsson and S. Steinthorsson, Hyperfine Interact., 1992, 70, 981-984. 